\title{
Kybernetika
}

\section{Giuseppina Barbieri}

A Dieudonné theorem for lattice group-valued measures

Kybernetika, Vol. 55 (2019), No. 5, 870-878

Persistent URL: http://dml.cz/dmlcz/147956

\section{Terms of use:}

(C) Institute of Information Theory and Automation AS CR, 2019

Institute of Mathematics of the Czech Academy of Sciences provides access to digitized documents strictly for personal use. Each copy of any part of this document must contain these Terms of use.

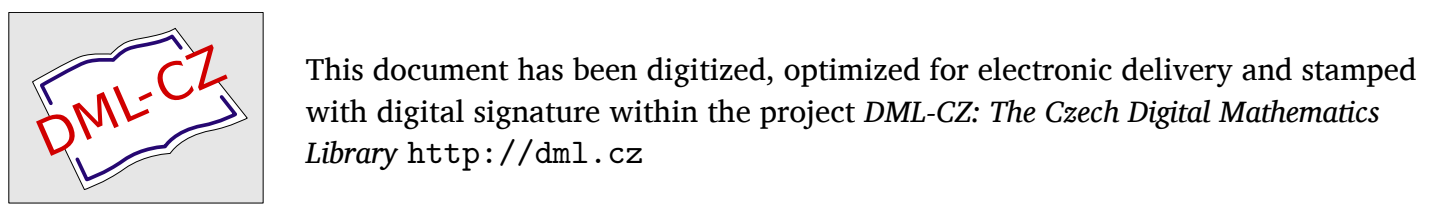




\title{
A DIEUDONNÉ THEOREM FOR LATTICE GROUP-VALUED MEASURES
}

\author{
Giuseppina BARBieri
}

A version of Dieudonné theorem is proved for lattice group-valued modular measures on lattice ordered effect algebras. In this way we generalize some results proved in the real-valued case.

Keywords: effect algebra, Dieudonné theorem, modular measures, lattice group

Classification: 28A12, 28A33, 28B15

\section{INTRODUCTION}

In 1933 Nikodým proved the well-known Vitali-Hahn-Saks theorem, namely if a sequence of Borel measures converges pointwise to a map $\mu$, then $\mu$ is a Borel measure. In 1951 Dieudonné proved the following more general theorem: "If a sequence of regular measures defined on Borel sets of a compact metrizable space converges on every open set, then it converges on every Borel sets. In this case, the sequence is uniformly regular". This theorem generalizes Nikodým's theorem if one substitutes the pointwise convergence on the Borel $\sigma$-algebra for the analogous condition on open sets provided a regularity assumption and a topological condition on the space are satisfied. In this note we furnish a general version of Dieudonné's theorem valid for lattice group-valued modular measures defined on lattice ordered effect algebras. For we use an abstract concept of convergence in lattice-group, namely $(D)$-convergence. Effect algebras (alias D-posets) have been independently introduced in 1994 by D. J. Foulis and M. K. Bennett in [4] and by F. Chovanec and F. Kôpka in [9] for modelling unsharp measurement in a quantum mechanical system. They are a generalization of many structures which arise in Quantum Physics [10] and in Mathematical Economics [8, 11, in particular they are a generalization of orthomodular posets and MV-algebras and therefore of Boolean algebras.

\section{PRELIMINARIES}

First we recall some basic facts on lattice groups.

DOI: $10.14736 /$ kyb-2019-5-0870 
Definition 2.1. Let $R$ be a lattice group.

$R$ is said to be Dedekind complete if every nonempty subset of $R$, bounded from above, has a supremum in $R$. We recall that a nonempty subset $S$ of $R$ bounded if there exists an element $u \in R$ with $|x| \leq u$ for every $x \in S$.

A sequence $\left(\sigma_{p}\right)_{p \in \mathbb{N}}$ in $R$ is an $(O)$-sequence if and only if it is decreasing and $\wedge_{p \in \mathbb{N}} \sigma_{p}=0$.

A bounded double sequence $\left(a_{t, l}\right)_{t, l}$ in $R$ is a $(D)$-sequence or a regulator if and only if $\left(a_{t, l}\right)_{l}$ is an $(O)$-sequence for any $t \in \mathbb{N}$. $R$ is weakly $\sigma$-distributive if and only if $\wedge_{\varphi \in \mathbb{N}^{\mathbb{N}}} \bigvee_{t=1}^{\infty} a_{t, \varphi(t)}=0$ for every $(D)$-sequence $\left(a_{t, l}\right)_{t, l}$ in $R$. A sequence $\left(x_{n}\right)$ in $R$ is order convergent (briefly, $(O)$-convergent) to $x$ if and only if there is a $(O)$-sequence $\left(\sigma_{p}\right)$ in $R$ such that for every $p \in \mathbb{N}$ there is $n_{0} \in \mathbb{N}$ with $\left|x_{n}-x\right| \leq \sigma_{p}$ whenever $n \geq n_{0}$ and we write $(O)-\lim x_{n}=x$.

A sequence $\left(x_{n}\right)$ in $R$ is $(O)$-Cauchy if and only if there is an $(O)$-sequence $\left(\tau_{p}\right)$ in $R$ such that for every $p \in \mathbb{N}$ there is $n_{0} \in \mathbb{N}$ with $\left|x_{n}-x_{q}\right| \leq \tau_{p}$ whenever $n, q \geq n_{0}$. A sequence $\left(x_{n}\right)$ in $R$ is $(D)$-convergent to $x$ if and only if there is a $(D)$-sequence $\left(a_{t, l}\right)$ in $R$ such that for every $\varphi \in \mathbb{N}^{\mathbb{N}}$ there is $n_{0} \in \mathbb{N}$ with $\left|x_{n}-x\right| \leq \bigvee_{t=1}^{\infty}\left(a_{t, \varphi(t)}\right)$ whenever $n \geq n_{0}$ and we write $(D)-\lim x_{n}=x$.

A sequence $\left(x_{n}\right)$ in $R$ is $(D)$-Cauchy if and only if there is a $(D)$-sequence $\left(b_{t, l}\right)_{t, l}$ in $R$ such that for every $\varphi \in \mathbb{N}^{\mathbb{N}}$ there is $n_{0} \in \mathbb{N}$ with $\left|x_{n}-x_{q}\right| \leq \bigvee_{t=1}^{\infty}\left(b_{t, \varphi(t)}\right)$ whenever $n, q \geq n_{0}$.

$R$ is said to be $(O)$-complete (respectively, $(D)$-complete) if and only if every $(O)$ Cauchy (respectively $(D)$-Cauchy) sequence is $(O)$-convergent (respectively, $(D)$-convergent).

Remark 2.2. Every Dedekind complete lattice group is both $(O)$-complete and $(D)$ complete. Moreover, every $(O)$-convergent sequence is also $(D)$-convergent to the same limit, the converse is true if and only if $R$ is weakly $\sigma$-distributive.

We recall the Fremlin lemma which is fundamental in what follows.

Lemma 2.3. ([13, Lemma 1C], [14, Theorem 3.2.3]) Let $R$ be Dedekind complete and $\left(a_{t, l}^{n}\right)_{t, l}, n \in \mathbb{N}$, be a sequence of regulators in $R$. Then for every $u \in R, u \geq 0$ there is a $(D)$-sequence $\left(a_{t, l}\right)_{t, l}$ in $R$ with

$$
u \wedge\left(\sum_{n=1}^{q} \bigvee_{t=1}^{\infty} a_{t, \varphi(t+n)}^{n}\right) \leq \bigvee_{t=1}^{\infty} a_{t, \varphi(t)}
$$

for every $q \in \mathbb{N}$ and $\varphi \in \mathbb{N}^{\mathbb{N}}$.

Now we define a $D$-lattice, or in other words a lattice ordered effect algebra.

From now on, let $R$ be a Dedekind complete lattice group.

Definition 2.4. Let $(L, \leq)$ be a poset with a smallest element 0 and a greatest element 1 and let $\ominus$ be a partial operation on $L$ such that $b \ominus a$ is defined if and only if $a \leq b$ and for all $a, b, c \in L$ :

If $a \leq b$ then $b \ominus a \leq b$ and $b \ominus(b \ominus a)=a$. 
If $a \leq b \leq c$ then $c \ominus b \leq c \ominus a$ and $(c \ominus a) \ominus(c \ominus b)=b \ominus a$.

Then $(L, \leq, \ominus)$ is called a difference poset (D-poset for short), or a difference lattice (D-lattice for short) if $L$ is a lattice.

One defines in $L$ a partial operation $\oplus$ as follows:

$a \oplus b$ is defined and $a \oplus b=c$ if and only if $c \ominus b$ is defined and $c \ominus b=a$.

The operation $\oplus$ is well-defined by the cancellation law [10, page 13] ( $a \leq b, c$ and $b \ominus a=c \ominus a$ implies $b=c)$, and $(L, \oplus, 0,1)$ is an effect algebra (see [10, Theorem 1.3.4]), that is the following conditions are satisfied for all $a, b, c \in L$ :

If $a \oplus b$ is defined, then $b \oplus a$ is defined and $a \oplus b=b \oplus a$;

If $b \oplus c$ is defined and $a \oplus(b \oplus c)$ is defined, then $a \oplus b$ and $(a \oplus b) \oplus c$ are defined, and $a \oplus(b \oplus c)=(a \oplus b) \oplus c$;

There exists a unique $a^{\prime} \in E$ such that $a \oplus a^{\prime}$ is defined and $a \oplus a^{\prime}=1$;

If $a \oplus 1$ is defined, then $a=0$.

We say that $a$ and $b$ are orthogonal if $a \leq b^{\prime}$ and we write $a \perp b$. Therefore $a \oplus b$ is defined if and only if $a \perp b$, and in this case $a \oplus b=\left(a^{\prime} \ominus b\right)^{\prime}$ by [10, Lemma 1.2.5].

Definition 2.5. A $D$-lattice $L$ is called $\sigma$-complete if it is closed under countable suprema and infima.

Proposition 2.6. Assume that $a, b, c$ are elements of an effect algebra $L$. Then

(i) If $a \leq b \leq c$, then $b \ominus a \leq c \ominus a$ and $(c \ominus a) \ominus(b \ominus a)=c \ominus b$

(ii) $a \ominus a=0, a \ominus 0=a$ and $a \oplus 0=a$

(iii) If $a \leq b$, then $b \ominus a=0$ if and only if $b=a$

(iv) If $a \leq b$, then $b=a \oplus(b \ominus a)$

(v) If $a \perp b$, then $a \leq a \oplus b$ and $(a \oplus b) \ominus a=b$

(vi) If $a \leq b \leq c^{\prime}$, then $a \oplus c \leq b \oplus c$

(vii) If $a \perp b$ and $a \perp c$, then $a \oplus b=a \oplus c$ if and only if $b=c$

(viii) If $a \leq b \leq c$, then $a \perp c \ominus b$ and $a \oplus(c \ominus b)=c \ominus(b \ominus a)$

(ix) If $L$ is a $D$-lattice, $\leq c$ and $b \leq c$, then $c \ominus(a \wedge b)=(c \ominus a) \vee(c \ominus b)$

From now on, let $L$ be a $D$-lattice.

Our source of information about $D$-lattices is the book by Dvurečenskij and Pulmannova [10]. 
Definition 2.7. A lattice group-valued map $\mu$ on $L$ is called a measure if it satisfies

$$
\mu(a \oplus b)=\mu(a)+\mu(b)
$$

whenever $a, b$ are orthogonal elements of $L$.

By [12] a modular function $\mu$ on a lattice is a map which satisfies

$$
\mu(a \vee b)+\mu(a \wedge b)=\mu(a)+\mu(b) .
$$

Definition 2.8. A lattice group-valued map $\mu$ on $L$ is called a modular measure it it is both a modular function and a measure.

The semivariation of $\mu$ is defined by

$$
\tilde{\mu}(a)=\bigvee\{|\mu(b)|: b \in L, b \leq a\}
$$

where $a \in L$.

For the rest of the paper, let $\mathcal{F}, \mathcal{G} \subseteq L$ be two lattices closed under the sum and $g \ominus f \in \mathcal{G}$ for each $f \in \mathcal{F}$ and $g \in \mathcal{G}$.

We make use of an abstract concept of regularity where $\mathcal{F}$ and $\mathcal{G}$ play the role of compact sets and open sets, respectively. (See Definition 3.3.

\section{REGULARITY AND EXHAUSTIVITY}

Definition 3.1. A lattice group-valued modular measure $\mu$ on $L$ is called $\mathcal{G}$-exhaustive if for every orthogonal sequence $\left(g_{n}\right)$ in $\mathcal{G}$ we have $(D)-\lim _{n} \mu\left(g_{n}\right)=0$

Let $\left(\mu_{i}\right)$ be a sequence of lattice group-valued modular measures on $L$. It is called uniformly $\mathcal{G}$-exhaustive if for for every orthogonal sequence $\left(g_{n}\right)$ in $\mathcal{G}$ we have $(D)$ $\lim _{n} \mu_{i}\left(g_{n}\right)=0$ uniformly with respect to $i \in \mathbb{N}$; more precisely, there is a $(D)$-sequence $\left(a_{t, l}\right)_{t, l}$ such that for every $\varphi \in \mathbb{N}^{\mathbb{N}}$ and $j \in \mathbb{N}$ there exists $n_{0} \in \mathbb{N}$ with $\tilde{\mu}_{j}\left(g_{n}\right) \leq$ $\vee_{t=1}^{\infty} a_{t, \varphi(t)}$ for every $n \geq n_{0}$.

Proposition 3.2. A lattice group-valued measure $\mu$ on $L$ is exhaustive if and only if every increasing sequence is a Cauchy sequence.

Proof. Let $\left(a_{n}\right)$ be an orthogonal sequence in $L$. Set $b_{n}=\oplus_{i \leq n} a_{i}$. Then $b_{n}$ is an increasing sequence, hence $(D)-\lim _{n} \mu\left(b_{n}\right)=0$. In particular, there is a $(D)$-sequence $\left(a_{t, l}\right)_{t, l}$ such that for every $\varphi \in \mathbb{N}^{\mathbb{N}}$ there exists $n_{0} \in \mathbb{N}$ with $\mu\left(b_{n+1} \ominus b_{n}\right) \leq \vee_{t=1}^{\infty} a_{t, \varphi(t)}$ for every $n \geq n_{0}$. Then $b_{n+1}=b_{n} \oplus\left(b_{n+1} \ominus b_{n}\right)$. On the other hand, we have $b_{n+1}=b_{n} \oplus a_{n}$, therefore $a_{n}=b_{n+1} \ominus b_{n}$. Hence $(D)-\lim _{n} \mu\left(a_{n}\right)=0$.

We now prove that every increasing sequence is Cauchy. By way of contradiction, let $\left(a_{n}\right)$ be an increasing sequence in $L$ and suppose that it is not Cauchy. Then we can inductively obtain subsequences $\left(b_{n}\right)$ and $\left(c_{n}\right)$ of $\left(a_{n}\right)$ with $b_{n} \leq c_{n} \leq b_{n+1}$ and for every $D$-sequence $a_{t, l}$ there are $\varphi \in \mathbb{N}^{\mathbb{N}}$ and $n \in \mathbb{N}$ with $\mu\left(c_{n} \ominus b_{n}\right) \not \leq \vee_{t=1}^{\infty} a_{t, \varphi(t)}$. Set $\left(d_{1}, d_{2}, \ldots d_{n}, \ldots\right)=\left(b_{1}, c_{1}, b_{2}, c_{2}, \ldots\right)$. Then $\left(d_{n}\right)$ is not Cauchy, a contradiction. 
Definition 3.3. A lattice group-valued modular measure $\mu$ on $L$ is regular if it satisfies the following properties:

(i) For every $a \in L$ there exists a couple of sequences $g_{n} \downarrow$ in $\mathcal{G}$ and $f_{n} \uparrow$ in $\mathcal{F}$ with

$$
f_{n}<f_{n+1}<a<g_{n+1}<g_{n} \text { for every } n \in \mathbb{N}
$$

and

$$
(D)-\lim \tilde{\mu}\left(g_{n} \ominus f_{n}\right)=0 .
$$

(ii) For every $b \in \mathcal{F}$ there exists a couple of sequences $g_{n} \downarrow$ in $\mathcal{G}$ and $f_{n} \downarrow$ in $\mathcal{F}$ with

$$
b<f_{n+1}<g_{n}<f_{n} \text { for every } n \in \mathbb{N}
$$

and

$$
(D)-\lim \tilde{\mu}\left(g_{n} \ominus b\right)=0 \text {. }
$$

Let $\left(\mu_{i}\right)$ be a sequence of lattice group-valued modular measures. We say that it is uniformly regular if the same above holds for the map $\sup _{i} \tilde{\mu}_{i}$; more precisely, if it satisfies the following properties:

(i) For every $a \in L$ there exists a couple of sequences $g_{n} \downarrow$ in $\mathcal{G}$ and $f_{n} \uparrow$ in $\mathcal{F}$ with

$$
f_{n}<f_{n+1}<a<g_{n+1}<g_{n} \text { for every } n \in \mathbb{N}
$$

and there is a $(D)$-sequence $\left(a_{t, l}\right)_{t, l}$ such that for every $\varphi \in \mathbb{N}^{\mathbb{N}}$ and $j \in \mathbb{N}$

$$
\text { there exists } n_{0} \in \mathbb{N} \text { with } \sup _{i} \tilde{\mu}_{i}\left(g_{n} \ominus f_{n}\right) \leq \vee_{t=1}^{\infty} a_{t, \varphi(t)} \text { for every } n \geq n_{0} \text {. }
$$

(ii) For every $b \in \mathcal{F}$ there exists a couple of sequences $g_{n} \downarrow$ in $\mathcal{G}$ and $f_{n} \downarrow$ in $\mathcal{F}$ with

$$
b<f_{n+1}<g_{n}<f_{n} \text { for every } n \in \mathbb{N}
$$

and there is a $(D)$-sequence $\left(a_{t, l}\right)_{t, l}$ such that for every $\varphi \in \mathbb{N}^{\mathbb{N}}$ and $j \in \mathbb{N}$

$$
\text { there exists } n_{0} \in \mathbb{N} \text { with } \sup _{i} \tilde{\mu}_{i}\left(g_{n} \ominus b\right) \leq \vee_{t=1}^{\infty} a_{t, \varphi(t)} \text { for every } n \geq n_{0} \text {. }
$$

\section{THE MAIN RESULTS}

We make use of the following theorem by Boccuto and Dimitriou:

Theorem 4.1. [7, Theorem 3.3] Let $E$ be a subset of a lattice $L$, satisfying property (E), and $m_{j}: L \rightarrow R$, be a sequence of equibounded finitely additive measures, whose restrictions on $E$ are exhaustive on $E$. If the limit (D)- $\lim _{j} m_{j}(e)$ exists in $R$ for every $e \in E$ with respect to a single regulator, then the $m_{j}$ s are uniformly exhaustive on $E$.

We recall that a lattice $E$ of subsets is said to satisfy property (E) iff every disjoint sequence $\left(C_{h}\right)_{h}$ in $E$ has a subsequence $\left(C_{h_{r}}\right)_{r}$, such that $E$ contains the $\sigma$-algebra generated by the sets $\left(C_{h_{r}}\right)_{r}$. 
Theorem 4.2. Suppose that $\mathcal{G}$ is closed under countable sums. Let $\left(\mu_{i}\right)$ be a sequence of equibounded pointwise convergent $\mathcal{G}$-exhaustive modular measures on $L$. Then $\left(\mu_{i}\right)$ is uniformly $\mathcal{G}$-exhaustive.

Proof. By way of contradiction there are strictly increasing sequence $i_{n}$ in $\mathbb{N}$ and an orthogonal sequence $\left(g_{n}\right)$ in $\mathcal{G}$ such that $(D)-\lim _{n} \mu_{i_{n}}\left(g_{n}\right) \neq 0$. Define $\nu_{n}(A):=$ $\mu_{i_{n}}\left(\oplus_{h \in A} g_{h}\right)$. With the aid of [2, 2.5] one can check that it is a sequence of finitely additive measures on the power set of $\mathbb{N}$. We can apply [7, Theorem 3.3]. So these restrictions form a uniformly exhaustive sequence. This contradicts the assumptions and it completes the proof.

Lemma 4.3. Suppose that $\left(\mu_{i}\right)$ is a sequence of equibounded regular uniformly $\mathcal{G}$ exhaustive modular measures on $L$. Let $b \in \mathcal{F}$ such that $(3)$ and $(4)$ hold for $\mu_{i}(i \in \mathbb{N})$. Then (6) holds true.

Proof. Let $a \in L$ with $a \leq g_{n} \ominus b$.

CLAIM: $\mu_{i}(a)=(D)-\lim _{m} \mu_{i}\left(a \ominus\left(f_{m} \ominus b\right) \wedge a\right)$.

PROOF: For every $m>n$ we have

$$
\mu_{i}(a)-\mu_{i}\left(a \ominus\left(f_{m} \ominus b\right) \wedge a\right)=\mu_{i}\left(\left(f_{m} \ominus b\right) \wedge a\right) \leq \mu_{i}\left(g_{m-1} \ominus b\right) \leq \tilde{\mu}_{i}\left(g_{m-1} \ominus b\right) .
$$

It suffices to show that $(D)-\lim _{n} \sup _{i} \tilde{\lambda}_{i}\left(g_{n} \ominus b\right)=0$, where $\lambda_{i}$ is the restriction of $\mu_{i}$ to $\mathcal{G}$. By way of contradiction this limit is different from 0 , then there exist $\left(e_{t, l}\right)_{t, l}$ a $(D)$-sequence and $\varphi \in \mathbb{N}^{\mathbb{N}}$ with the property that for every $p \in \mathbb{N}, i \in \mathbb{N}$, there exist $n>p, i \in \mathbb{N}$ and an element $a \in \mathcal{G}$ such that $a \leq g_{n} \ominus b$ and $\mu_{i}(a) \not \leq \vee_{t=1}^{\infty} e_{t, \varphi(t)}$ and so there exist $\left(e_{t, l}\right)_{t, l}$ a $(D)$-sequence and $\varphi \in \mathbb{N}^{\mathbb{N}}$ with the property that for every $p \in \mathbb{N}, i \in \mathbb{N}$, there exist $m>p, i \in \mathbb{N} \mu_{i}\left(a \ominus\left(f_{m} \ominus b\right) \wedge a\right) \not \leq \vee_{t=1}^{\infty} e_{t, \varphi(t)}$.

Hence we can construct by induction four sequences $\left(n_{k}\right),\left(i_{k}\right),\left(m_{k}\right)$ and $\left(a_{k}\right)$ in $\mathcal{G}$ with $a_{k} \leq g_{n_{k}} \ominus b \leq g_{n_{k-1}} \ominus b$ and $\left.\mu_{i}\left(a_{k} \ominus\left(f_{m_{k}} \ominus b\right) \wedge a_{k}\right)\right) \not \leq \vee_{t=1}^{\infty} e_{t, \varphi(t)}$. Since

$$
\left.g_{n_{k-1}} \ominus g_{m_{k}}=\left(g_{n_{k-1}} \ominus b\right) \ominus\left(g_{n_{k}} \ominus b\right) \geq\left(f_{m_{k}} \ominus b\right) \vee a_{k}\right) \ominus\left(f_{m_{k}} \ominus b\right) .
$$

We get

$$
\begin{gathered}
\mu_{i_{k}}\left(g_{n_{k-1}}\right)-\mu_{i_{k}}\left(g_{m_{k}}\right) \geq \mu_{i_{k}}\left(\left(f_{m_{k}} \ominus b\right) \vee a_{k}\right)-\mu_{i_{k}}\left(f_{m_{k}} \ominus b\right) \\
\left.=\mu_{i_{k}}\left(a_{k} \ominus\left(\left(f_{m_{k}} \ominus b\right) \wedge a_{k}\right)\right)\right)
\end{gathered}
$$

with $n_{k-1}<n_{k}<m_{k}$. As $\left(g_{n}\right)$ is a monotone sequence which is not a Cauchy sequence, this contradicts the uniform $\mathcal{G}$-exhaustivity of $\left(\mu_{i}\right)$.

Applying Lemma 4.3 for $\mathcal{F}=\mathcal{G}=L$ and $b=0$ we have:

Corollary 4.4. Let $\left(\mu_{i}\right)$ be a sequence of equibounded uniformly exhaustive modular measures on $L$ and let $\left(a_{n}\right)$ be a decreasing sequence of elements of $L$ such that $(D)$ $\lim _{n} \tilde{\mu}_{i}\left(a_{n}\right)=0$ for every $i \in \mathbb{N}$. Then the limit is uniformly with respect to $i \in \mathbb{N}$. 
Theorem 4.5. Let $\left(\mu_{i}\right)$ be a sequence of equibounded regular uniformly $\mathcal{G}$-exhaustive modular measures on $L$. Then it is uniformly exhaustive and uniformly regular.

Proof. We have to show that $\left(\mu_{i}\right)$ is uniformly regular. The last item defining the uniform regularity is fulfilled thanks to Lemma 4.3. For the first: Let $a \in L$ and $f_{n}, g_{n}$ satisfying (1) and (2). To prove (5), apply Corollary 4.4 to $a_{n}=g_{n} \ominus f_{n}$.

Now we prove that $\left(\mu_{i}\right)$ is uniformly exhaustive. By way of contradiction there are a sequence $\left(a_{n}\right)$ of orthogonal elements of $L$ and a sequence $i_{n}$ such that $(D)-$ $\lim _{n} \mu_{i_{n}}\left(a_{n}\right) \neq 0$. Thanks to the regularity we may suppose that $a_{n} \in \mathcal{F}$. Put $b_{n}=$ $a_{1} \oplus \cdots \oplus a_{n}$.

CLAIM: There exists a couple of sequences $g_{n} \uparrow$ in $\mathcal{G}$ and $f_{n} \uparrow$ in $\mathcal{F}$ such that $b_{n} \leq g_{n} \leq f_{n} \leq g_{n+1}$ and

$$
\bigvee_{i \in \mathbb{N}} \tilde{\mu}_{i}\left(f_{n} \ominus b_{n}\right) \leq \bigvee_{t=1}^{\infty} a_{t, \varphi(t+n)}^{n}
$$

for each $n \in \mathbb{N}$.

PROOF of the CLAIM: Proceed by induction.

(a) The base case: By uniform regularity we can pick $g_{1} \in \mathcal{G}, f_{1} \in \mathcal{F}$ with $b_{1} \leq g_{1} \leq f_{1}$ and

$$
\bigvee_{i \in \mathbb{N}} \tilde{\mu}_{i}\left(f_{1} \ominus b_{1}\right) \leq \bigvee_{t=1}^{\infty} a_{t, \varphi(t+1)}^{1} .
$$

(b) The inductive step: Again, by uniform regularity we can construct the $(h+1)$ th step in such a way $g_{h+1} \in \mathcal{G}, f_{h+1} \in \mathcal{F}$ with $b_{h+1} \vee f_{h} \leq g_{h+1} \leq f_{h+1}$ and

$$
\bigvee_{i \in \mathbb{N}} \tilde{\mu}_{i}\left(f_{h+1} \ominus\left(b_{h+1} \vee f_{h}\right)\right) \leq \bigvee_{t=1}^{\infty} a_{t, \varphi(t+h+1)}^{h+1}
$$

Thanks to Lemma 2.3 let $\left(a_{t, l}\right)_{t, l}$ be with

$$
u \wedge\left(\sum_{n=1}^{h} \bigvee_{t=1}^{\infty} a_{t, \varphi(t+n)}^{n}\right) \leq \bigvee_{t=1}^{\infty} a_{t, \varphi(t)}
$$

for every $h \in \mathbb{N}$ and $\varphi \in \mathbb{N}^{\mathbb{N}}$. Hence, for each $h \in \mathbb{N}$, as $f_{h+1} \ominus b_{h+1} \leq\left(f_{h+1} \ominus\left(b_{h+1} \vee\right.\right.$ $\left.\left.f_{h}\right)\right) \oplus\left(\left(b_{h+1} \vee f_{h}\right) \ominus b_{h+1}\right)$ and $\tilde{\mu}_{i}\left(\left(b_{h+1} \vee f_{h}\right) \ominus b_{h+1}\right) \leq \tilde{\mu}_{i}\left(f_{h} \ominus b_{h}\right)$ we get

$$
\tilde{\mu}_{i}\left(f_{h+1} \ominus b_{h+1}\right) \leq \tilde{\mu}_{i}\left(f_{h+1} \ominus\left(b_{h+1} \vee f_{h}\right)\right)+\tilde{\mu}_{i}\left(f_{h} \ominus b_{h}\right) \leq \vee_{t=1}^{\infty} a_{t, \varphi(t)}+\vee_{t=1}^{\infty} a_{t, \varphi(t)}
$$

for each $h \in \mathbb{N}$ and $\varphi \in \mathbb{N}^{\mathbb{N}}$.

Then we get $\tilde{\mu}_{i}\left(a_{n+1}\right)=\tilde{\mu}_{i}\left(b_{n+1} \ominus b_{n}\right) \leq \tilde{\mu}_{i}\left(g_{n+1} \ominus f_{n}\right)+\tilde{\mu}_{i}\left(f_{n} \ominus b_{n}\right)$ as

$$
b_{n+1} \ominus b_{n} \leq\left(g_{n+1} \ominus f_{n}\right) \oplus\left(f_{n} \ominus b_{n}\right) .
$$

Since by regularity $(D)-\lim _{n} \tilde{\mu}_{i}\left(g_{n+1} \ominus f_{n}\right)=0$, and by above $(D)-\lim _{n} \tilde{\mu}_{i}\left(f_{n} \ominus b_{n}\right)=0$, we get a contradiction with the hypothesis by absurd. 
Theorem 4.6. Let $\mathcal{G}$ be closed under countable sums. Let $\left(\mu_{i}\right)$ be a sequence of equibounded $\mathcal{G}$-exhaustive regular modular measures on $L$ converging on every element of $\mathcal{G}$. Then the sequence is converging on every element of $L$ and it is uniformly exhaustive. Therefore its limit is exhaustive and regular.

Pro of. Thanks to 4.2 the sequence is uniformly $\mathcal{G}$-exhaustive.

We shall show that for each element $a \in L$, the sequence $\left(\mu_{i}(a)\right)_{i \in \mathbb{N}}$ is a Cauchy sequence and then we can apply Theorem 4.5 to complete the proof. Let $f \in \mathcal{F}, g \in \mathcal{G}$ with $f \leq a \leq g$, then there exists a $(D)$-sequence $a_{t . l}$ such that $\sup \tilde{\mu}_{i}(g \ominus f) \leq \vee_{t=1}^{\infty} a_{t, \varphi(t)}$ for every $\varphi \in \mathbb{N}^{\mathbb{N}}$.

Now we shall use the following equality:

$$
\mu_{i}(a)-\mu_{j}(a)=\mu_{i}(g)-\mu_{j}(g)+\mu_{j}(g \ominus a)-\mu_{i}(g \ominus a) .
$$

Since $\mu_{i}(g)$ is a Cauchy sequence there exist a $(D)$-sequence $\left(b_{t . l}\right)_{t . l}$ and $n_{0} \in \mathbb{N}$ such that $\left|\mu_{i}(g)-\mu_{j}(g)\right| \leq \vee_{t=1}^{\infty} b_{t, \varphi(t)}$ for every $\varphi \in \mathbb{N}^{\mathbb{N}}$ and every $i, j \geq n_{0}$. Hence $d_{t . l}=a_{t . l}+b_{t . l}$ is a $(D)$-sequence. Therefore there exists $n_{0} \in \mathbb{N}$ such that $\mid \mu_{i}(a)-$ $\mu_{j}(a) \mid \leq \vee_{t=1}^{\infty} d_{t, \varphi(t)}$ for every $\varphi \in \mathbb{N}^{\mathbb{N}}$ and every $i, j \geq n_{0}$, whence the thesis follows.

(Received October 30, 2018)

\section{REFERENCES}

[1] A. Avallone: Separating points of measures on effect algebras. Mathematica Slovaca 20 (2006), 203-214.

[2] A. Avallone: Cafiero and Nikodym boundedness theorem in effect algebras. Ital. J. Pure Appl. Math. 57 (2007), 2, 129-140.

[3] G. Barbieri: On the Dieudonné theorem. Sci. Math. Japon. 70 (2009), 3, 279-284.

[4] M. K. Bennett and D. J. Foulis: Effect algebras and unsharp quantum logics. Special issue dedicated to Constantin Piron on the occasion of his sixtieth birthday. Found. Phys. 24 (1994), 10, 1331-1352. DOI:10.1007/bf02283036

[5] A. Boccuto: Dieudonné-type theorems for means with values in Riesz spaces. Tatra Mountains Math. Publ. 8 (1996), 9-42.

[6] A. Boccuto and D. Candeloro: Some new results about Brooks-Jewett and Dieudonnétype theorems in (1)-groups. Kybernetika 46 (2010), 6, 1049-1060.

[7] A. Boccuto and X. Dimitriou: Equivalence between limit theorems for lattice groupvalued $k$-triangular set functions. Mediterr. J. Math. 4 (2018), Art. 174, 20 pp. DOI:10.1007/s00009-018-1222-9

[8] D. Butnariu and E. P. Klement: Triangular norm-based measures and games with fuzzy coalitions. Theory and Decision Library. Series C: Game Theory, Mathematical Programming and Operations Research, 10. Kluwer Academic Publishers Group, Dordrecht 1993. DOI:10.1007/978-94-017-3602-2

[9] F. Chovanec and F. Kôpka: D-posets. Math. Slovaca 44 (1994), 1, 21-34.

[10] A. Dvurečenskij and S. Pulmannová: New Trends in Quantum Structures. Kluwer Academic Publishers, Bratislava 2000. DOI:10.1007/978-94-017-2422-7_1 
[11] L. G. Epstein and J. Zhang: Subjective probabilities on subjectively unambiguous events. Econometrica 69 (2001), 2, 265-306. DOI:10.1111/1468-0262.00193

[12] I. Fleischer and T. Traynor: Equivalence of group-valued measures on an abstract lattice. Bull. Acad. Polon. Sci. Sci. Math. 28 (1980), 11-12, 549-556.

[13] D. H. Fremlin: A direct proof of the Matthes-Wright integral extension theorem. J. London Math. Soc. 11 (1975), 2, 276-284.

[14] B. Riečan and T. Neubrunn: Integral, Measure and Ordering. Kluwer Acad. Publ./Ister Science, Dordrecht/Bratislavia 1997. DOI:10.1007/978-94-015-8919-2

Giuseppina Barbieri, University of Salerno.

e-mail: gibarbieri@unisa.it 